\title{
Thermal effects in Stokes' Second Problem for Unsteady Second Grade Fluid Flow through Porous Medium
}

\author{
Dr. K. Ramakrishna Reddy ${ }^{1}$, Dr.B. Rama Bhupal Reddy ${ }^{2}$ \\ ${ }^{1}$ Department of Mathematics, SRIT, Ananthapuramu, A.P., India \\ ${ }^{2}$ Department of Mathematics, KSRMCE, Kadapa-516003, A.P., India
}

\begin{abstract}
In this paper, we studied the effect of thermal in Stokes' second problem for unsteady second grade fluid flow through porous medium. The expressions for the velocity field and the temperature field are obtained analytically. The effects of various emerging parameters on the velocity field and temperature field are studied through graphs in detail.
\end{abstract}

Keywords: Thermal Effects, Fluid Flow, Porous Medium

\section{Introduction}

There has been an increase in interest in the effect of porous media, because of their extensive practical applications in geophysics, thermal insulation in buildings, petroleum resources, packed-bed reactors and sensible heat-storage beds. Many studies related to non-Newtonian fluids saturated in a porous medium have been carried out. Dharmadhikariand Kale [5] studied experimentally the effect of non-Newtonian fluids in a porous medium. Chen and Chen [4] investigated the free convection flow along a vertical plate embedded in a porous medium. Rees [17] analyzed the effect of inertia on free convection over a horizontal surface embedded in a porous medium. Nakayama [13] investigated the effect of buoyancy-induced flow over a non-isothermal body of arbitrary shape in a fluid-saturated porous medium. A ray-tracing method for evaluating the radiative heat transfer in a porous medium was examined by Argento[1].

Recently, the study of non-Newtonian fluids has attracted much attention because of their practical applications. With the growing importance of non-Newtonian fluids in modern technology and industries, investigations of such fluids are desirable. A number of industrially important fluids including molten plastics, polymers, pulps, foods and fossil fuels, which may saturate in underground beds areexhibits non-Newtonian behavior. Due to complexity of fluids, several non-Newtonian fluid models have been proposed. In the category of such fluids, second grade fluid is the simplest subclassfor which one can hope to gain an analytic solution. Exact analytic solutions for the flows of non-Newtonian fluids aremost welcome provided they correspond to physically realistic situations, as they serve a dual purpose. First, they providea solution to flow that has technical relevance. Second, such solutions can be used as checks against complicatednumerical codes that have been developed for much more complex flows. Various studies on the flows of non-Newtonianfluids have been made under different physical aspects. However some recent contributions in the field may bementioned in Refs. (Fetecau and Fetecau, [9]; Hayat et al., [11]; Chen et al., [3]; Fetecau and Fetecau, [10]; Tan and Masuoka, [19]).

The motion of a viscous fluid caused by the sinusoidal oscillation of a flat plate is termed as Stokes' second problem by Schliching[18]. Initially, both the plate and fluid are assumed to be at rest. At time $\mathrm{t}=0+$, the plate suddenly starts oscillating with the velocity $U_{0} e^{i \omega t}$. The study of the flow of a viscous fluid over an oscillating plate is not only of fundamental theoretical interest but it also occurs in many applied problems such as acoustic streaming around an oscillating body, an unsteady boundary layer with fluctuations etc(Tokuda,[20]). Penton [14] has presented a closed-form to the transient component of the solution for the flow of a viscous fluid due to an oscillating plate. Puri and Kythe[15] have discussed an unsteady flow problem which deals with non-classical heat conduction effects and the structure of waves in Stokes' second problem. Erdogan[8] analyzed the unsteady flow of viscous fluid due to an oscillating plane wall by using Laplace transform technique. Vajravelu and Rivera [21] discussed the hydromagnetic flow at an oscillating plate. Much work has been published on the flow of fluid over an oscillating plate for different constitutive models (Erdogan, [7]; ZengandWeinbaum, [22]; Puri and Kythe,[15]; Asghar et al., [2]; Ibrahem et al., [12].

\section{Mathematical Formulation}

We consider the one-dimensional unsteady flow of a laminar, incompressible second grade fluid through a porous medium past a vertical flat plate in the $y z$-plane and occupy the space $x>0$, with $x$-axis in the vertical direction. The plate initially at rest and at constant temperature $\theta_{\infty}$ which is the free stream 
temperature is moved with a velocity $U_{0} e^{i \omega t}$ in its own plane along the z-axis, and its temperature is subjected to a periodic heating of the form $\left(\theta_{w}-\theta_{\infty}\right) e^{i \omega t}$, where $\theta_{w} \neq \theta_{\infty}$ is some constant.

Viscoelastic fluids can be modeled by Rivlin - Ericksen constitutive equation

$$
\mathrm{S}=-p \mathrm{I}+\mu \mathrm{A}_{1}+\alpha_{1} \mathrm{~A}_{2}+\alpha_{2} \mathrm{~A}_{1}^{2}
$$

where $\mathrm{S}$ is the Cauchy stress tensor, $p$ is the scalar pressure, $\mu, \alpha_{1}$ and $\alpha_{2}$ are the material constants, customarily known as the coefficients of viscosity, elasticity and cross - viscosity, respectively. These material constants can be determined from viscometric flows for any real fluid. $A_{1}$ and $A_{2}$ are Rivlin-Ericksen tensors and they denote, respectively, the rate of strain and acceleration. $\mathrm{A}_{1}$ and $\mathrm{A}_{2}$ are defined by

$$
\mathrm{A}_{1}=\nabla \mathrm{V}+(\nabla \mathrm{V})^{T}
$$

and

$$
\mathrm{A}_{2}=\frac{d \mathrm{~A}_{1}}{d t}+\mathrm{A}_{1}(\nabla \mathrm{V})+(\nabla \mathrm{V})^{T} \mathrm{~A}_{1}
$$

where $d / d t$ is the material time derivative and $\nabla$ gradient operator and ()$^{T}$ transpose operator. The viscoelastic fluids when modeled by Rivlin-Ericksen constitutive equation are termed as second-grade fluids. A detailed account of the characteristics of second - grade fluids is well documented by Dunn and Rajagopal[6]. Rajagopal and Gupta [16] have studied the thermodynamics in the form of dissipative inequality (Clausius Duhem) and commonly accepted the idea that the specific Helmholtz free energy should be a minimum in equilibrium. From the thermodynamics consideration they assumed

$$
\mu \geq 0, \quad \alpha_{1}>0, \quad \alpha_{1}+\alpha_{2}=0
$$

We seek the velocity field of the form

$$
(u(x, t), 0,0)
$$

For this type of flow,equation of continuity is identically satisfied and the balance of linear momentum reduces to the following differential equation (Fetecauand Fetecau [10])

$$
\rho \frac{\partial u}{\partial t}=\mu \frac{\partial^{2} u}{\partial x^{2}}+\alpha_{1} \frac{\partial^{3} u}{\partial x^{2} \partial t}-\frac{\mu}{k} u+\rho g \beta\left(\theta-\theta_{0}\right)
$$

The energy equation (MCF model) is given by (Ibrahem et al.,[12])

$$
\tau \theta_{t t}+\theta_{t}=\frac{\chi}{\rho c_{p}} \theta_{x x}
$$

Introducing the following non dimensional variables

$$
\bar{x}=\frac{u_{0}}{v} x, \quad \bar{u}=\frac{u}{u_{0}}, \bar{t}=\frac{u_{0}^{2}}{v} t, \quad \bar{\theta}=\frac{\theta-\theta_{0}}{\theta_{w}-\theta_{0}}
$$

into the Eqs. (6) and (7), we get

$$
\begin{gathered}
\frac{\partial u}{\partial t}=\frac{\partial^{2} u}{\partial x^{2}}+\alpha \frac{\partial^{3} u}{\partial x^{2} \partial t}+G \theta-\frac{1}{D a} u \\
p \lambda \frac{\partial^{2} \theta}{\partial t^{2}}+p \frac{\partial \theta}{\partial t}=\frac{\partial^{2} \theta}{\partial x^{2}} \\
\text { where } \alpha=\frac{\alpha_{1} u_{0}^{2}}{\mu \nu}, D a=\frac{k u_{0}^{2}}{v^{2}}, G=\frac{v g \beta\left(\theta_{w}-\theta_{0}\right)}{U_{0}^{3}}, p=\frac{v \rho c_{p}}{\psi}, \lambda=\frac{\tau U_{0}^{2}}{v} .
\end{gathered}
$$

The corresponding dimensions are boundary conditions are

$$
\begin{array}{ll}
u(0, t)=e^{i \omega t}, \theta(0, t)=e^{i \omega t} \\
u(\infty, t)=0, & \theta(\infty, t)=0
\end{array}
$$




\section{Solution}

To solve the non-linear system (3) and (4) using the boundary conditions (10),we assume that $u(x, t)=U(x) e^{i \omega t}, \theta(x, t)=\Theta(x) e^{i \omega t}$

Substituting Eq. (11) intoEqs.(3)and(4) and the boundary conditions (10), we get

$$
\begin{aligned}
& \frac{d^{2} U}{d x^{2}}-m^{2} U=-G n e^{-k x} \\
& \frac{d^{2} \Theta}{d x^{2}}+\left(\lambda p \omega^{2}-i \omega p\right) \Theta=0
\end{aligned}
$$

here $m^{2}=\frac{\frac{1}{D a}+\omega^{2} \alpha+i \omega\left(1-\frac{\alpha}{D a}\right)}{1+\omega^{2} \alpha^{2}}$ and $n=\frac{1-i \omega \alpha}{1+\omega^{2} \alpha^{2}}$.

The boundary conditions are

$$
\begin{aligned}
& U(0)=1, \Theta(0)=1 \\
& U(\infty)=0, \Theta(\infty)=0
\end{aligned}
$$

Solving the equations (12) - (13) using the boundary conditions Eq. (14), we obtain $\theta=e^{-k x}$

$U=e^{-m x}+\frac{G n}{k^{2}-m^{2}}\left[e^{-m x}-e^{-k x}\right]$

where $k=\sqrt{-\lambda p \omega^{2}+i \omega p}=\sqrt{\omega P\left(\frac{\sqrt{\omega^{2} \lambda^{2}+1}-\lambda \omega}{2}\right)}+i \sqrt{\omega P\left(\frac{\sqrt{\omega^{2} \lambda^{2}+1}+\lambda \omega}{2}\right)}$.

\section{Results and Discussion}

Figs. 1 - 8 show the effects of various values of the emerging parameters $\alpha, G, p$ and $D a$ on the velocity ( $\operatorname{Re} u$ and $|u|)$ profiles.

Fig. 1shows the effects of material parameter $\alpha$ on $\operatorname{Re} u$ for $D a=0.1, \quad p=1$, $\omega=10, t=0.1, \lambda=0.005$ and $G=5$. It is found that, the $\operatorname{Re} u$ decreases with increasing $\alpha$. The same trend is observed from Fig. 2 for $|u|$.Fig. 3 depicts the effects of $G$ on $\operatorname{Re} u$ for $D a=0.1, p=1$, $\omega=10, t=0.1, \lambda=0.005$ and $\alpha=0.01$. It is observed that, the $\operatorname{Re} u$ initially increases and then decreases with increasing $G$. Effects of $G$ on $|u|$ for $D a=0.1, \quad p=1, \quad \omega=10, \quad t=0.1$, $\lambda=0.005$ and $\alpha=0.01$ is shown in Fig. 4. It is noted that, the $|u|$ increases with an increase in $G$.

Fig. 5 shows the effects of $D a$ on $\operatorname{Re} u$ for $G=5, p=1, \omega=10, t=0.1, \lambda=0.005$ and $\alpha=0.01$. It is found that, the $\operatorname{Re} u$ first increases and then decreases with increasing $D a$. Fig. 6 represents the effects of $D a$ on $|u|$ for $G=5, p=1, \omega=10, t=0.1, \lambda=0.005$ and $\alpha=0.01$. It is observed that, the $|u|$ increases with an increase in $D a$.Effects of $p$ on $\operatorname{Re} u$ for $G=5, D a=0.1, \omega=10$, $t=0.1, \lambda=0.005$ and $\alpha=0.01$ is shown in Fig. 7. It is found that, the $\operatorname{Re} u$ first decreases and then increasing with increasing $p$.Effects of $p \quad$ on $|u|$ for $\quad G=5, \quad D a=0.1, \quad \omega=10$, $t=0.1, \lambda=0.005$ and $\alpha=0.01$ is depicted in Fig. 8. It is noted that, the $|u|$ decreases on increasing $p$.Fig. 9 shows the effects of $\lambda$ on $\operatorname{Re} \theta$ for $G=5, p=1, \omega=10, t=0.1, \quad D a=0.1$ and $\alpha=0.01$. It is observed that, the $\operatorname{Re} \theta$ first increases and then decreases with increasing $\lambda$. Fig. 
10depictstheeffects of $\lambda$ on $|\theta|$ for $G=5, p=1, \omega=10, t=0.1, D a=0.1$ and $\alpha=0.01$. It is noted that, the $|\theta|$ increases with an increase in $\lambda$.

Effects of $p$ on $\operatorname{Re} \theta$ for $G=5, \lambda=0.005, \omega=10, t=0.1, D a=0.1$ and $\alpha=0.01$ is depicted in Fig. 11. It is found that, the $\operatorname{Re} \theta$ first decreases and then increases with an increase in $p$.Fig. 12 illustrates the effects of $p$ on $|\theta|$ for $G=5, \lambda=0.005, \omega=10, t=0.1, D a=0.1$ and $\alpha=0.01$. It is observed that, the $|\theta|$ decreases with increasing $p$.

\section{Conclusions}

We studied the effect of thermal in Stokes second problem for unsteady second grade fluid flow through porous medium. The expressions for the velocity field and the temperature field are obtained analytically. It is found that, the $\operatorname{Re} u$ first decreases and then increases with increasing $\alpha$ or $p$, while it first decreases and then decreases with increasing $G$ or $D a$. Further it is observed that, the $|u|$ decreases with increasing $\alpha$ or $p$, while it increases with increasing $G$ or $D a$. Also, it is observed that, $\operatorname{Re} \theta$ first increases and then decreases with increasing $\lambda$, while $\operatorname{Re} \theta$ first decreases and then increases with increasing $p$. Further, it is found that, the $|\theta|$ increases with increasing $\lambda$, whereas it decreases with increasing $p$.

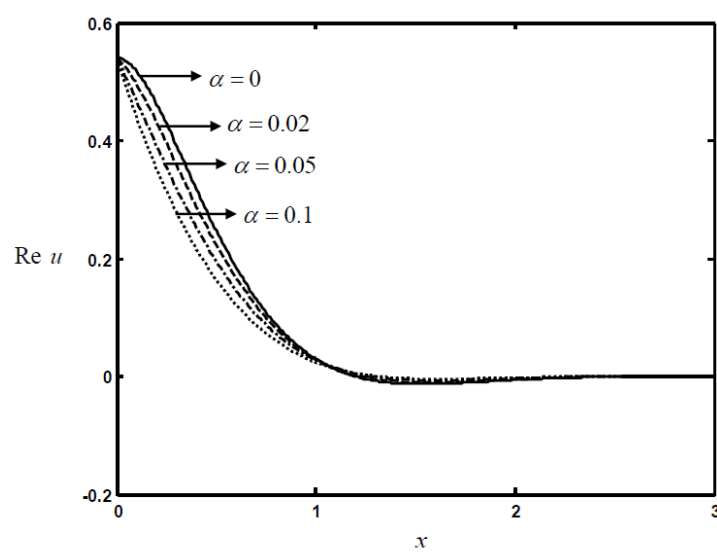

Fig. 1. Effects of $\alpha$ on $\operatorname{Re} u$ for $D a=0.1$, $p=1, \omega=10, t=0.1, \lambda=0.005$ and $G=5$.

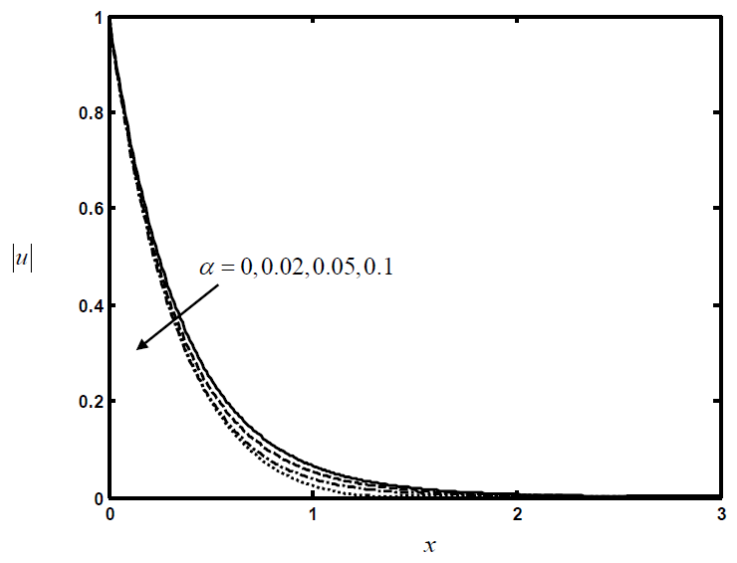

Fig. 2 Effects of $\alpha$ on $|u|$ for $D a=0.1, p=1$, $\omega=10, t=0.1, \lambda=0.005$ and $G=5$. 


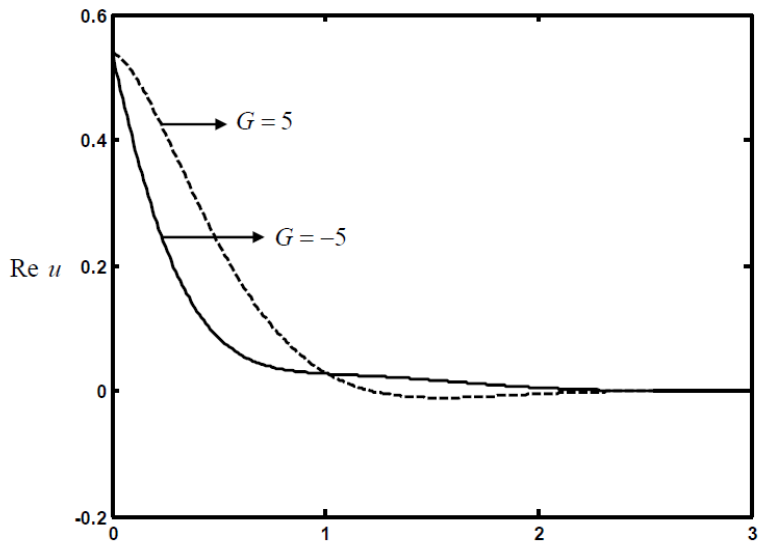

Fig. 3. Effects of $G$ on $\stackrel{x}{\operatorname{Re}} u$ for $D a=0.1$, $p=1, \quad \omega=10, \quad t=0.1, \quad \lambda=0.005$ and $\alpha=0.01$.

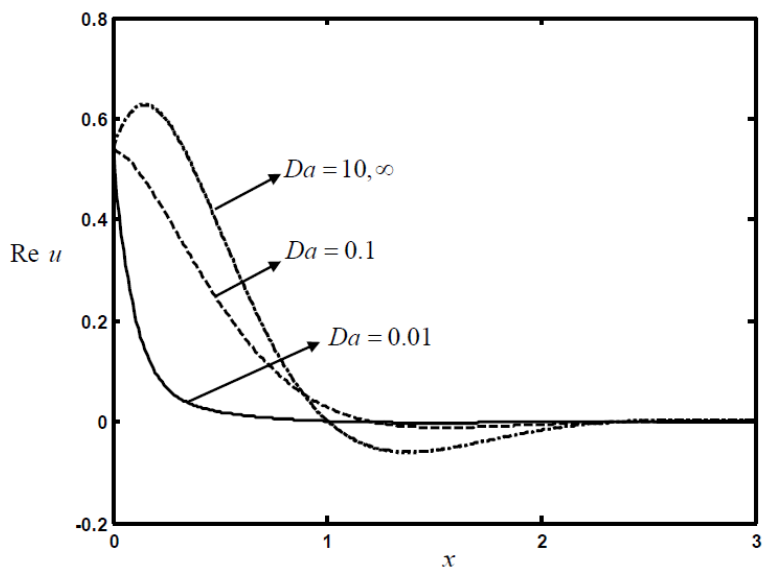

Fig. 5. Effects of $D a$ on $\operatorname{Re} u$ for $G=5, p=1$, $\omega=10, t=0.1, \lambda=0.005$ and $\alpha=0.01$.

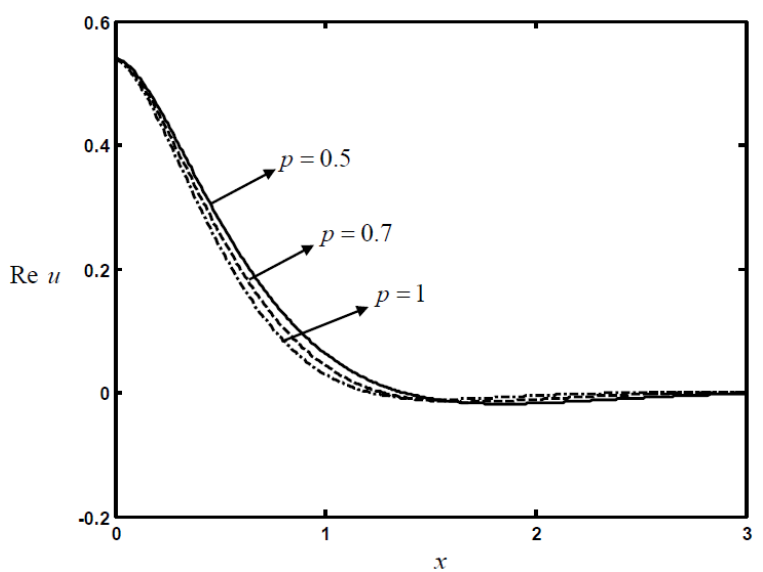

Fig. 7. Effects of $p$ on $\operatorname{Re} u$ for $G=5$,

$D a=0.1, \omega=10, t=0.1, \lambda=0.005$ and $\alpha=0.01$.

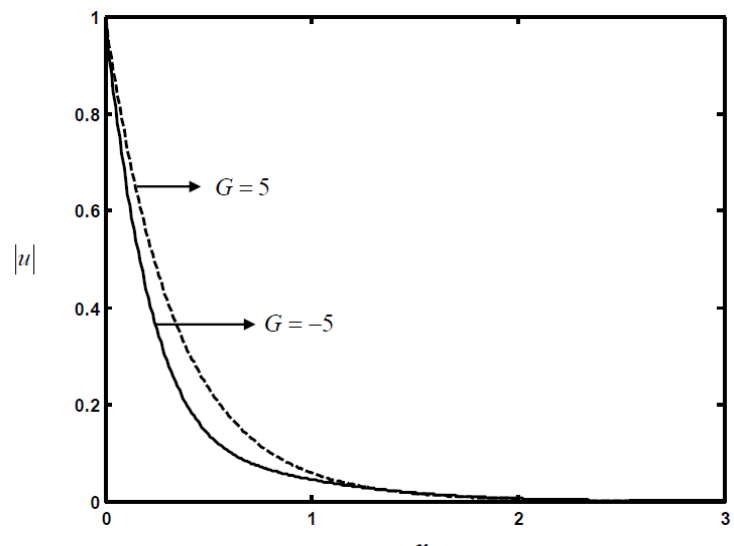

Fig. 4. Effects of $G$ on $|u|$ for $D a=0.1, p=1$, $\omega=10, t=0.1, \lambda=0.005$ and $\alpha=0.01$.

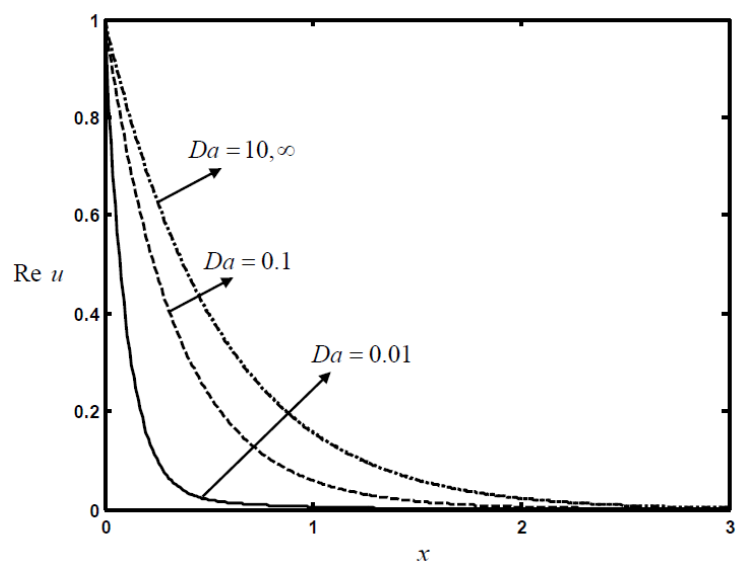

Fig. 6. Effects of $D a$ on $|u|$ for $G=5, p=1$, $\omega=10, t=0.1, \lambda=0.005$ and $\alpha=0.01$

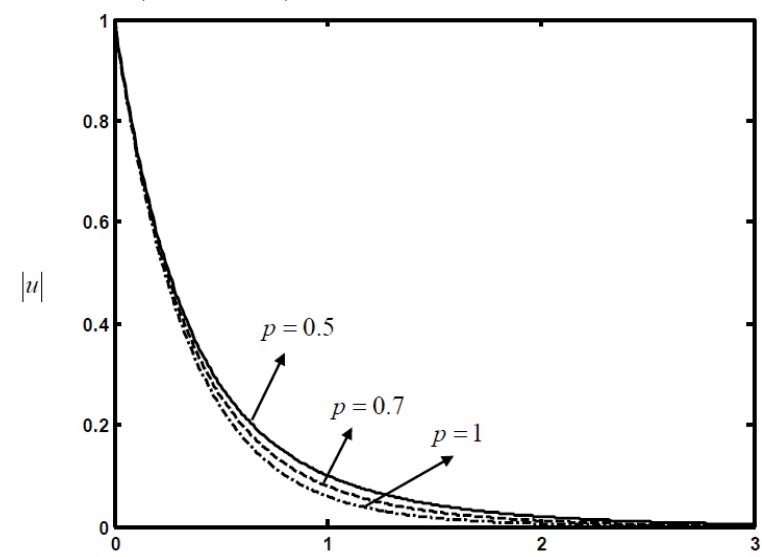

Fig. 8. Effects of $p$ on $|u|$ for $G=5, D a=0.1$, $\omega=10, t=0.1, \lambda=0.005$ and $\alpha=0.01$. 


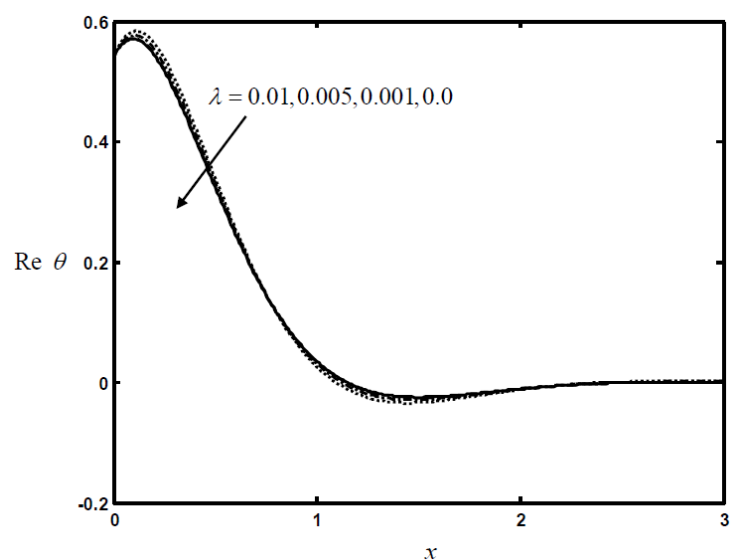

Fig.9. Effects of $\lambda$ on $\operatorname{Re} \theta$ for $G=5, p=1$, $\omega=10, t=0.1, D a=0.1$ and $\alpha=0.01$.

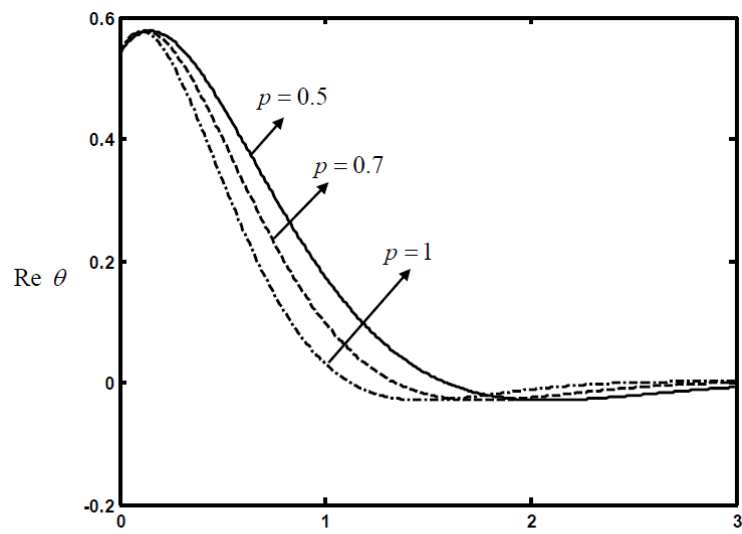

Fig.11. Effects of $p$ on $\operatorname{Re} \theta$ for $G=5$, $\lambda=0.005, \omega=10, t=0.1, D a=0.1$ and $\alpha=0.01$

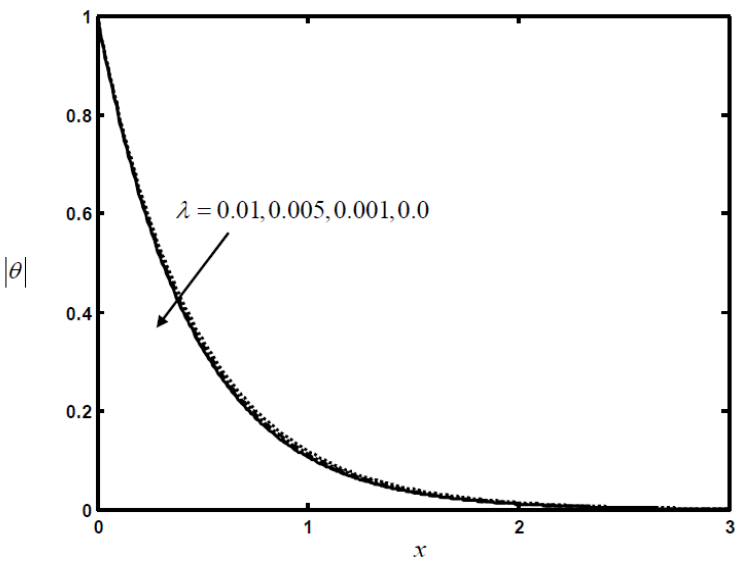

Fig. 10. Effects of $\lambda$ on $|\theta|$ for $G=5, p=1$, $\omega=10, t=0.1, D a=0.1$ and $\alpha=0.01$.

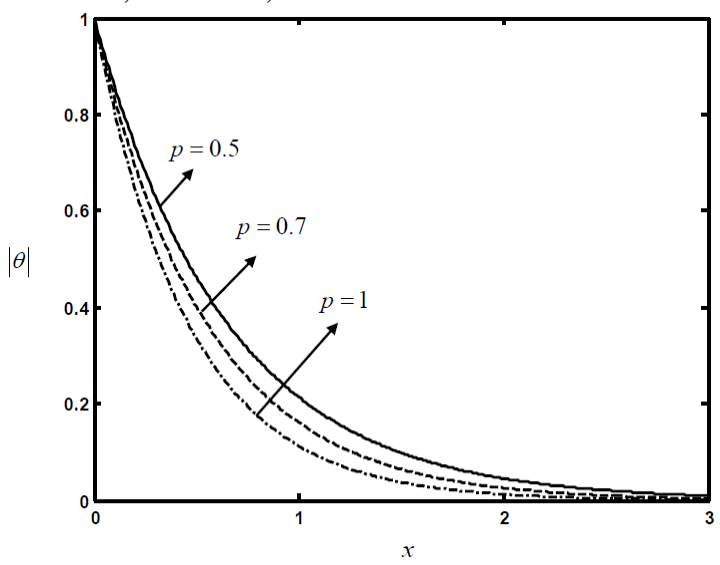

Fig. 12. Effects of $p$ on $|\theta|$ for $G=5$, $\lambda=0.005, \omega=10, t=0.1, D a=0.1$ and $\alpha=0.01$.

\section{References}

[1] C. Argento and D. Bouvard, A Ray Tracing Method for Evaluating the Radiative Heat Transfer in Porous Medium", Int. J. Heat Mass Transfer, 39(1996),3175-3180.

[2] S. Asghar, T. Hayat and A.M. Siddiqui, Moving boundary in a non-Newtonian fluid, Int. J. Nonlinear Mech., 37(2002), 75-80.

[3] C.I. Chen, C.K. Chen and Y.T. Yang, Unsteady unidirectional flow of an Oldroyd-B fluid in a circular duct with different given volume flow rate conditions. Heat and Mass Transfer, 40(2004), 203-209.

[4] H. Chen and C. Chen, Free convection flow of non-newtonian fluids along a vertical plate embedded in a porous medium, Journal of Heat Transfer, 110(1988), 257-260.

[5] R.V. Dharmadhikari,andD.D.Kale, Flow of non-newtonian fluids through porous media, Chem. Eng. Sci., 40(1985), 527-529.

[6] J.E. Dunn and K.R. Rajagopal, Fluids of differential type: critical review and thermodynamic analysis, Int. J. Engng. Sci., 33(1995), 689-729.

[7] M. E. Erdogan, Plane surface suddenly set in motion in a non-Newtonian fluid, Acta Mech.,108(1995), 179-187.

[8] M. E. Erdogan, A note on an unsteady flow of a viscous fluid due to an oscillating plane wall, Int. J. Non-Linear Mech., 35(2000), $1-6$.

[9] C. Fetecau and C. Fetecau, A new exact solution for the flow of Maxwell fluid past an infinite plate, Int. J. Non-Linear Mech.,38 (2003), 42-37.

[10] C.Fetecau and C.Fetecau. Starting solutions for some unsteady unidirectional flows of a second grade fluid, Int. J. Engng. Sci., 43(2005), 781-789.

[11] T. Hayat, Y. Wang and K. Hutter, Hall effects on the unsteady hydromagnetic oscillatory flow of a second grade fluid. Int. J. Non-Linear Mech.,39(2004),1027-37.

[12] F. S. Ibrahem, I. A. Hassanien and A. A. Bakr, Thermal effects in Stokes' second problem for unsteady micropolar fluids flow, Applied Mathematics and Comput., 173(2006), 916 -937.

[13] A. Nakayama and H. Koyama, Buoyancy-induced flow of non-newtonian fluids over a non-isothermal body of arbitrary shape in a porous medium, Applied Scientific Research, 48(1991), 55-70.

[14] R. Penton, The transient for Stokes' oscillating plane: a solution in terms of tabulated functions, J. Fluid Mech., 31(1968), 819825.

[15] P. Puri, P.K. Kythe, Thermal effects in Stokes' second problem, Acta Mech., 112(1998), 44-50. 
[16] K. R. Rajagopal and A. S.Gupta. An exact solution for the flow of a non-Newtonian fluid past an infinite porous plate, Mecanica, 19(1984), 158-160.

[17] D.A.S. Ress, The Effect of Inertia on Free Convection from a Horizontal Surface Embedded ina Porous Medium", Int. J. Heat Mass Transfer, 39 (1996), (1996), 3425-3430.

[18] H. Schlichting, K. Gersten, Boundary Layer Theory, 8th edition, Springer, Berlin, 2000.

[19] W. C.Tan, T. Masuoka, Stokes first problem for second grade fluid in a porous half space. Int. J. Non-Linear Mech., 40(2005), $515-522$.

[20] N. Tokuda, On the impulsive motion of a flat plate in a viscous fluid, J. Fluid Mech. 33(1968), 657-672.

[21] K. Vajravelu, J. Rivera, Hydromagnetic flow at an oscillating plate, Int. J. Non-Linear Mech.,38(2003), 305-312.

[22] Y. Zeng and S. Weinbaum, Stokes' problem for moving half planes, J. Fluid Mech., 287(1995), 59-74. 